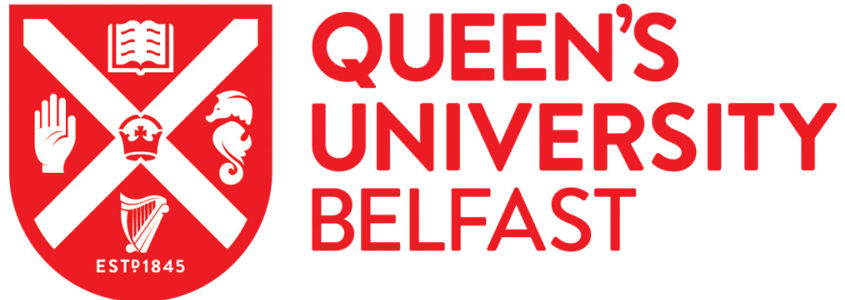

\section{An analysis of geometric uncertainty calculations for prostate radiotherapy in clinical practice.}

McGarry, C. K., Cosgrove, V. P., Fleming, V. A. L., O'Sullivan, J., \& Hounsell, A. R. (2009). An analysis of geometric uncertainty calculations for prostate radiotherapy in clinical practice. British Journal of Radiology, 82(974), 140-147. https://doi.org/10.1259/bjr/20582161

Published in:

British Journal of Radiology

Document Version:

Publisher's PDF, also known as Version of record

Queen's University Belfast - Research Portal:

Link to publication record in Queen's University Belfast Research Portal

Publisher rights

(c) 2009 The British Institute of Radiology

\section{General rights}

Copyright for the publications made accessible via the Queen's University Belfast Research Portal is retained by the author(s) and / or other copyright owners and it is a condition of accessing these publications that users recognise and abide by the legal requirements associated with these rights.

Take down policy

The Research Portal is Queen's institutional repository that provides access to Queen's research output. Every effort has been made to ensure that content in the Research Portal does not infringe any person's rights, or applicable UK laws. If you discover content in the Research Portal that you believe breaches copyright or violates any law, please contact openaccess@qub.ac.uk. 


\title{
An analysis of geometric uncertainty calculations for prostate radiotherapy in clinical practice
}

\author{
${ }^{1} \mathrm{C} K$ MCGARRY, MSc, ${ }^{1} \mathrm{~V}$ P COSGROVE, PhD, ${ }^{2} \mathrm{~V}$ A L FLEMING, PgD, ${ }^{2,3} \mathrm{~J} \mathrm{M}$ O'SULLIVAN, FFRRCSI and \\ ${ }^{1}$ A R HOUNSELL, PhD
}

\begin{abstract}
${ }^{1}$ Northern Ireland Regional Medical Physics Agency, ${ }^{2}$ Department of Clinical Oncology, Northern Ireland Cancer Centre, Belfast City Hospital Trust, Lisburn Road, Belfast BT9 7AB and ${ }^{3}$ Centre for Cancer Research and Cell Biology, Queens University, Belfast, Northern Ireland, UK
\end{abstract}

\begin{abstract}
Margins are used in radiotherapy to assist in the calculation of planning target volumes. These margins can be determined by analysing the geometric uncertainties inherent to the radiotherapy planning and delivery process. An important part of this process is the study of electronic portal images collected throughout the course of treatment. Set-up uncertainties were determined for prostate radiotherapy treatments at our previous site and the new purpose-built centre, with margins determined using a number of different methods. In addition, the potential effect of reducing the action level from $5 \mathrm{~mm}$ to $3 \mathrm{~mm}$ for changing a patient set-up, based on off-line bony anatomy-based portal image analysis, was studied. Margins generated using different methodologies were comparable. It was found that set-up errors were reduced following relocation to the new centre. Although a significant increase in the number of corrections to a patient's set-up was predicted if the action level was reduced from $5 \mathrm{~mm}$ to $3 \mathrm{~mm}$, minimal reduction in patient set-up uncertainties would be seen as a consequence. Prescriptive geometric uncertainty analysis not only supports calculation and justification of the margins used clinically to generate planning target volumes, but may also best be used to monitor trends in clinical practice or audit changes introduced by new equipment, technology or practice. Simulations on existing data showed that a $3 \mathrm{~mm}$ rather than a $5 \mathrm{~mm}$ action level during off-line, bony anatomy-based portal imaging would have had a minimal benefit for the patients studied in this work.
\end{abstract}

Received 5 December 2007 Revised 25 January 2008 Accepted 14 February 2008

DOI: $10.1259 / \mathrm{bjr} / 20582161$

(C) 2009 The British Institute of Radiology
Accurate definition of target volumes is fundamental to the radiotherapy treatment planning process. Generally, a margin is added to the gross tumour volume (GTV) to account for subclinical disease and so create a clinical target volume (CTV). A further margin is then added to account for geometric uncertainties, including patient set-up errors, to create the planning target volume (PTV). A description of the relationship between these different volumes is presented in International Commission on Radiation Units and Measurements (ICRU) 50 and 62 publications [1, 2].

Following the ICRU recommendations, a number of authors have proposed methodologies for determining target volume margins. Van Herk et al [3] proposed a method in which the margins ensure that $90 \%$ of patients receive an equivalent uniform dose (EUD) of at least $98 \%$ (known as the Van Herk method from here on). In 2003, the British Institute of Radiology (BIR) published "Geometric Uncertainties in Radiotherapy: Defining the Planning Target Volume" [4], in which the source of many geometric uncertainties were identified and used to

Address correspondence to: C K McGarry, Northern Ireland Regional Medical Physics Agency, Northern Ireland Cancer Centre, Belfast City Hospital Trust, Lisburn Road, Belfast BT9 7AB, Northern Ireland, UK. E-mail: Conor.McGarry@mpa.n-i. nhs.uk generate margins in order that the CTV is covered in $90 \%$ of cases (known as the BIR method from here on). A number of techniques for generating the required margins from geometric uncertainties have been the subject of review [5].

Part of the dataset necessary to determine geometric uncertainties, and hence the required margins, can be obtained using electronic portal images (EPIs) of the treatment fields. By comparing the digitally reconstructed radiograph (DRR), calculated from the therapy CT scan, with the EPI data, an assessment of the accuracy of each delivered field can be made [6]. Processes designed to support the systematic analysis and review of patient set-up in this way have been described in the literature $[7,8]$.

It has been reported [4] that online imaging and reactive patient repositioning during radiotherapy can significantly improve treatment accuracy and reproducibility, and potentially lead to smaller margins around the target volume. There is, however, a requirement that the set-up errors, both random and systematic, are evaluated. It is likely that the set-up errors will vary depending upon the treatment site and technique, immobilization equipment used, departmental practice, and even the age and quality of the imaging and treatment machines being used. Once established, these data can be used both as a measure of the quality of the 
treatment delivery at any one time, and as a means to monitor and confirm changes introduced by the use of new equipment or treatment techniques.

The aim of this work is to find set-up accuracies at our previous site (Belvoir Park Hospital (BPH)), and then at the new Northern Ireland Cancer Centre (NICC) following transfer of services in March 2006. We report on the application of the BIR method [4] to determine margins for conformal prostate radiotherapy and compared the results with those using the Van Herk method [3].

Finally, to investigate how the set-up uncertainties may be improved, and potentially lead to a reduction in target volume margins, the portal imaging data gathered were used to predict the impact of a $3 \mathrm{~mm}$, rather than the current $5 \mathrm{~mm}$, action level on the calculated set-up uncertainties.

\section{Methods and background}

\section{Relocation of the clinical radiation oncology service}

In March 2006, the Northern Ireland radiation oncology clinical service was relocated from BPH to a new purpose-built facility at the NICC. This saw a significant replacement and upgrade in radiotherapy equipment, including linear accelerators, simulators and imaging equipment. However, for the patients undergoing conformal external-beam prostate radiotherapy in this study, the main treatment parameters were identical at both sites. This included (i) treatment position (supine), (ii) immobilization (knee rest and ankle stocks), (iii) planned beam arrangement (a three-field class solution anterior and a pair of parallel-opposed wedged laterals), (iv) prescription (70 Gy in 35 fractions using $15 \mathrm{MV}$ photons) and (v) portal imaging protocol.

At $\mathrm{BPH}$, all patients were imaged on a Siemens Emotion 6 CT scanner (Siemens Medical Systems, Forcheim, Germany) with $5 \mathrm{~mm}$ spaced slices and planned on a Theraplan Plus (TPP) v3.8 (Nucletron BV, Veenendal, the Netherlands) external beam treatment planning system. Portal images for 25 patients were analysed, after acquisition using an aS500 amorphous silicon electronic portal imaging device (EPID) on one of two Varian 2100CD Clinacs (Varian Medical Systems, Palo Alto, CA). Before treatment, all plans were verified on a Simulix simulator (Nucletron BV.).

At the NICC, patients underwent CT scans either on the Siemens Emotion 6 (moved from BPH) or on a new GE Lightspeed RT CT scanner (GE Medical Systems, Milwaukee, WI), again using $5 \mathrm{~mm}$ spaced slices. Planning is now performed on an Oncentra MasterPlan (OMP) v1.4sp4 system (Nucletron B.V.). Portal images for a further 25 patients were analysed, having been acquired using an aS500 EPID on one of two newer Varian 2100CD Clinacs. Before treatment, plans were verified on an Acuity simulator (Varian Medical Systems Inc.).

\section{Portal imaging}

The portal imaging protocol implemented at both sites is as follows: anteroposterior (AP) and left-right/right- left lateral double-exposed electronic portal images (EPIs) are acquired during the first three fractions of the treatment course (days 1-3), and then AP and lateral EPIs are acquired on a weekly basis. Both laterals were imaged on day 1 and then alternated between left-right and right-left on each subsequent imaging day. A $2 \mathrm{~cm}$ margin was applied to the treatment field jaw settings for the double-exposed part of the image. Each EPI was compared with the DRR generated from the therapy CT scans, using Varis v7 (Varian Medical Systems Inc.) offline image matching tools. An action level of $5 \mathrm{~mm}$ was defined for this process. A shift in the patient set-up instructions was applied when a systematic deviation greater than the $5 \mathrm{~mm}$ action level was observed between the DRR and portal images, and would be applied following delivery of the first three fractions. If a set-up offset greater than $5 \mathrm{~mm}$ was observed during the weekly image analysis, a repeat image was scheduled; if the offset was reproduced, a correction was applied to this set-up (an average of the weekly displacement and the repeat displacement).

\section{Determination of geometric uncertainties}

\section{BIR 2003: equation and related uncertainties}

The PTV as described by ICRU [1, 2] is a geometrical concept. It is defined by taking into consideration the net effect of all the possible geometrical variations in order to ensure that the prescribed dose is actually delivered to the CTV [1]. The BIR method for calculating a CTV-PTV margin [4] separates errors into those introduced through the treatment preparation stage, in which errors will be propagated systematically through the course of the treatment, and the treatment execution errors that result from the day-to-day uncertainties of set-up, organ shape and motion. This method also takes into account the effect of different treatment beam configurations.

The systematic Gaussian error $(\Sigma)$ is defined as being composed of the doctor delineation error, motion error, phantom transfer error and systematic set-up error, summed in quadrature. A random Gaussian error (treatment execution error $-\sigma$ ) is composed of the random set-up error, motion error and beam penumbra, summed in quadrature. The total CTV-PTV margin is given by:

$$
\mathrm{CTV}-\mathrm{PTV} \text { margin }=2.5 \sum+a+b+\beta\left(\sigma-\sigma_{\mathrm{p}}\right)
$$

where $\Sigma$ is the systematic error, $\sigma$ is the treatment execution error, $\beta$ is the planning parameter (to account for different treatment beam configurations), $\sigma_{p}$ is the beam penumbra width, $a$ is the linear treatment planning beam algorithm error and $b$ is the linear breathing error (i.e. the uncertainties to the target position associated with a patient's respiration cycle).

The magnitude of the uncertainty in delineating the target is related to the imaging modality used and the level of expertise in interpreting the image and outlining the target. Within the BIR (2003) handbook, it was concluded that the "gold standard" imaging modality was MRI alongside CT. With these modalities, it was 
proposed that, for prostate radiotherapy treatments, a doctor delineation error of $2 \mathrm{~mm}$ in all directions could be used. For this work, we reviewed values presented in the literature and applied the value used by Rasch et al (in 1999) [9] on the basis that it most closely follows what happens in clinical practice in our centre.

Prostate motion error has been studied by a large number of authors [10-13] and was discussed in detail in the BIR (2003) handbook [4]. A review by Langen and Jones [10] showed variability between studies, although they reported that prostate motion was generally of the same magnitude for each study: $0.6 \pm 0.7 \mathrm{~mm}$, $-0.4 \pm 3.9 \mathrm{~mm}$ and $-0.2 \pm 3.2 \mathrm{~mm}$ for lateral, AP and superior-inferior motion, respectively. The values attained by the BIR handbook were used for the motion error.

Set-up errors can be determined by systematic analysis of EPIs and DRRs. It is recommended in the BIR handbook that information from at least 20 patients should be used. Set-up errors may range from $1.0-4.0 \mathrm{~mm}$ [14]. In this work, imaging data from the two sets of 25 patients were analysed, so giving 50 sets of 19 EPIs that were matched to the reference DRRs. Offsets in the left, right, superior, inferior, anterior and posterior directions were each recorded individually. From these measurements, the standard deviation of the systematic set-up error and mean standard deviation of treatment set-up errors were determined. Portal images show combined effects of setup errors and phantom transfer error, but not delineation error or organ motion error, because the organ is not visible in the portal image. The calculation in the BIR handbook reflects this by including the systematic set-up error but not the transfer error within the combined systematic set-up error. The calculations for our centres, both BPH and NICC, also assumes that the phantom transfer error is constant and contained within the systematic set-up error, as each dataset was collected over a relatively short period of time.

An action level was specified at $5 \mathrm{~mm}$. This produces a top hat function for the distribution of set-up errors, with a corresponding standard deviation of $1 / \sqrt{3}$ times the action level $(1 / \sqrt{3} \times 5 \mathrm{~mm}=2.9 \mathrm{~mm}$ in our case $)$. However, on analysis of the changes made to the patient's set-up instructions in this study, it was identified that the actual action level being observed was closer to $4 \mathrm{~mm}$. This was because, whenever systematic uncertainties were revealed to be $4 \mathrm{~mm}$, the patient's set-up was routinely corrected at the clinician's discretion. Therefore, an action level of $4 \mathrm{~mm}$ with a corresponding standard deviation of $4 \mathrm{~mm} / \sqrt{3}$ for the top hat distribution function was used in the analysis, as it better described the protocol used in clinical practice.

The treatment planning system (TPS) algorithm error is a linear systematic uncertainty. If the width between the high-dose levels in the penumbrae of treatment beams calculated and displayed by the TPS is larger than that delivered, there will be a systematic under-dosing of the CTV and PTV. Therefore, the margin around the CTV should be increased to account for this. Water tank profile measurements of a $15 \mathrm{MV}$ photon beam with a field size of $10 \times 10 \mathrm{~cm}^{2}$ at $10 \mathrm{~cm}$ deep with a source to skin distance (SSD) of $90 \mathrm{~cm}$, taken during commissioning, were used to find this parameter. The measured profile widths at the $90 \%$ dose level were compared with calculations generated on the treatment planning systems.
The magnitude of the beam penumbra will have an effect on the field size required and the probability of CTV coverage. The photon penumbra error may be measured by dividing the distance between the $10 \%$ and the $90 \%$ level of a single beam at a typical treatment depth by 2.56. (This is derived by assuming that the penumbra dose is described by an error function.) Profile measurements of a $15 \mathrm{MV}$ photon beam with a field size of $10 \times 10 \mathrm{~cm}^{2}$ at $10 \mathrm{~cm}$ deep with SSD $90 \mathrm{~cm}$ and at $20 \mathrm{~cm}$ deep with SSD $80 \mathrm{~cm}$, taken during commissioning, were used to find this parameter.

The planning parameter, which accounts for the effect that the number of beams and geometry can have on overall beam penumbra, was provided by the BIR (2003) handbook for the class solution of treatment beams used at our centre (anterior and two wedged lateral beams).

Although it has been suggested that patients' breathing may influence prostate movement during a radiotherapy treatment [15], it is possible that it is related to the immobilization technique and equipment used [16]. Therefore, this parameter was set to zero for all calculations in this study.

\section{Van Herk method: equation and related uncertainties}

A second method for determining geometric uncertainties was also investigated. This method is simpler than the BIR method and has been used in a number of studies [17-19]. Like the BIR method, it uses the combined systematic error and the treatment execution error but does not consider the penumbra, breathing, motion, planning parameter or the algorithm error explicitly. The equation is given by:

$$
\mathrm{CTV}-\mathrm{PTV} \text { margin }=2.5 \sum+0.7 \sigma
$$

This margin ensures that $90 \%$ of patients have an EUD of $98 \%$. The delineation and prostate motion errors (SI, AP and lateral) were defined by Rasch et al [9] and Van Herk et al [20]. The set-up uncertainties were taken from measurements made by Bel et al [21].

\section{Tolerance reduction}

Further analysis of the NICC data was performed with respect to the $5 \mathrm{~mm}$ action level used. First, any corrections that had been made to a patient set-up were removed from the EPI data so that it represented the daily set-up without a correction strategy in place [22]. Each patient dataset was then re-analysed and corrected based on a $3 \mathrm{~mm}$ action level (i.e. whenever a systematic displacement was greater than $3 \mathrm{~mm}$, a correction was applied to all subsequent data points). If the remaining data points revealed a systematic set-up that exceeded the action level, again all subsequent data points were corrected. Although this does not replicate exactly the process that would be used in practice (a weekly "out of tolerance" set-up would require an image acquisition the following day to confirm a systematic set-up variation), it does provide an estimate of the effect of size of the action level on the set-up uncertainties. It can be used to help predict any increase in the number of changes to the 
patient's set-up during the course of their treatment as a consequence of this lower action level.

\section{Results}

\section{Geometric uncertainties used to generate margins}

All uncertainties used in the calculations are given in Table 1. These include the planning parameter, the breathing error, the doctor delineation error and the motion error. The value obtained for the algorithm error (a) for the TPP system was $-1.5 \mathrm{~mm}$, whereas for the OMP system it was $-0.7 \mathrm{~mm}$. These values are negative because the $90 \%$ isodoses calculated were less than those measured in the water tank. At $10 \mathrm{~cm}$ and $20 \mathrm{~cm}$ deep, the measured $90-10 \%$ penumbra was $11 \mathrm{~mm}$ and $16 \mathrm{~mm}$, respectively. Therefore, the average photon penumbra width $\left(\sigma_{\mathrm{p}}\right.$; beam penumbra divided by the 2.56 error function) used was rounded to $5.0 \mathrm{~mm}$, as this closely follows the value used in the BIR 2003 handbook.

\section{Magnitude of uncertainties}

Table 2 illustrates the set-up uncertainties calculated in this work, and are given along with values calculated in the BIR (2003) handbook and those reported by Enmark et al [19]. The reasons for using this publication were that it was recent, it used CT for delineation and it closely followed how patients are set up at our centre (using external markers).

Figure 1 shows histograms comparing the calculated uncertainties at BPH and those at NICC. There is more spread seen for BPH than NICC, which is reflected in the larger set-up uncertainties seen in Table 2.

\section{Treatment margins}

Table 3 shows the required CTV-PTV margin calculated for the two sites using the BIR method, along with given values from the literature. It should be noted that, in the BIR 2003 example, MRI was used to delineate the prostate and all delineation uncertainties were set to $2 \mathrm{~mm}$. At our centre, (and in Enmark et al [19]), CT alone is used and therefore the values given in Table 1 were used as the doctor delineation error.

Table 4 shows the CTV-PTV margins generated using the Van Herk method, along with examples from the literature for comparison; where applicable, the same measured uncertainties were used.
Table 5 shows the possible effects that different correction strategies would have on generating CTVPTV margins using the BIR method. The correction strategy with a protocol of no correction was simulated by replacing any shifts applied through the original $5 \mathrm{~mm}$ correction strategy and then using the resulting set-up errors (Table 2) to generate the margins. All other components in the equation were unchanged. The set-up errors from the simulated $3 \mathrm{~mm}$ action level in Table 2 were also used to generate margins for comparison (Table 5). Also shown are the margins generated had there been no (zero) set-up errors.

\section{Discussion}

It can be seen from Table 2 that the random errors were consistent for both BPH and NICC patients; as identical techniques were used to set up patients for imaging and treatment (i.e. immobilization, use of skin tattoos), this was expected. However, there appears to be a reduction in the calculated systematic error and this improvement is more clearly demonstrated in Figure 1. The data provide evidence that the transfer of the clinical service was achieved without detriment to the accuracy of the radiotherapy treatment. Analysis of the geometric accuracy of radiotherapy treatment was prudent, as more than one device (CT scanners, treatment planning system and linear accelerators) changed following the move to the new radiotherapy centre. The reduction in the systematic error may be a consequence of the use of new imaging and treatment delivery equipment.

The uncertainties reported here are similar to those quoted in the BIR 2003 handbook and are lower than those quoted by Enmark et al [19] for conformal radiotherapy. Hurkmans et al [14] suggest that random and systematic set-up errors of $2.5 \mathrm{~mm}$ can be considered to be "state-of-the art" for prostate treatment techniques.

Table 3 lists the CTV-PTV margins calculated from the uncertainties when following the BIR method. Although an improvement was observed in systematic set-up errors for the NICC data, this did not lead to a significant reduction in the margin for all orientations. One of the reasons for this was that the TPP algorithm error had a larger effect in reducing the margin. In addition, the doctor delineation error and motion errors are dominant in the creation of these margins. This is demonstrated in Table 5 by setting the set-up uncertainties to zero and calculating the margins using the uncertainties in Table 1. The greatest margin reduction due to a reduc-

Table 1. Geometric uncertainties obtained from the literature [referenced] or measured (M) using a $15 \mathrm{MV} 10 \times 10 \mathrm{~cm}^{2} \mathrm{photon}$ beam

\begin{tabular}{lccc}
\hline Uncertainty & Lateral $(\mathrm{mm})$ & Superior-inferior $(\mathrm{mm})$ & Anteroposterior $(\mathrm{mm})$ \\
\hline Doctor [9] & 1.7 & 2.8 & 2.0 \\
Motion [4] & 1.0 & 2.5 & 3.0 \\
Breathing [4] & 0 & 0 & 0 \\
Phantom transfer [4] & 3.0 & 3.0 & 3.0 \\
Planning parameter [4] & 1.04 & 1.64 & 1.44 \\
Algorithm TPP/OMP (M) & $-1.5 /-0.7$ & $-1.5 /-0.7$ & $-1.5 /-0.7$ \\
Penumbra (M) & 5.0 & 5.0 & 5.0 \\
\hline
\end{tabular}

TPP, Theraplan Plus; OMP, Oncentra MasterPlan. 


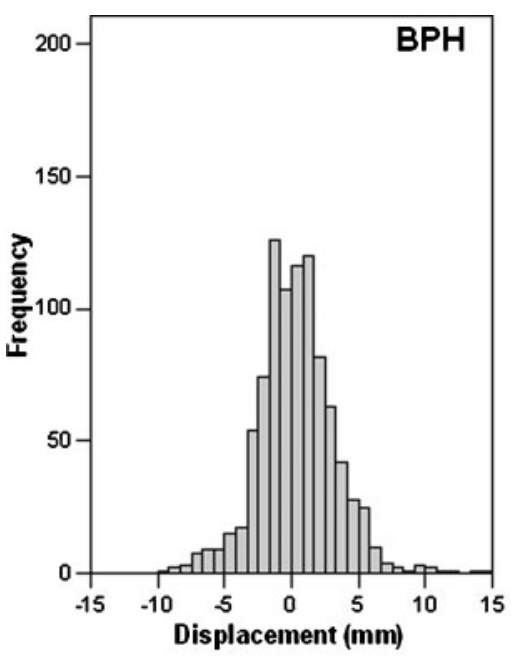

(a)

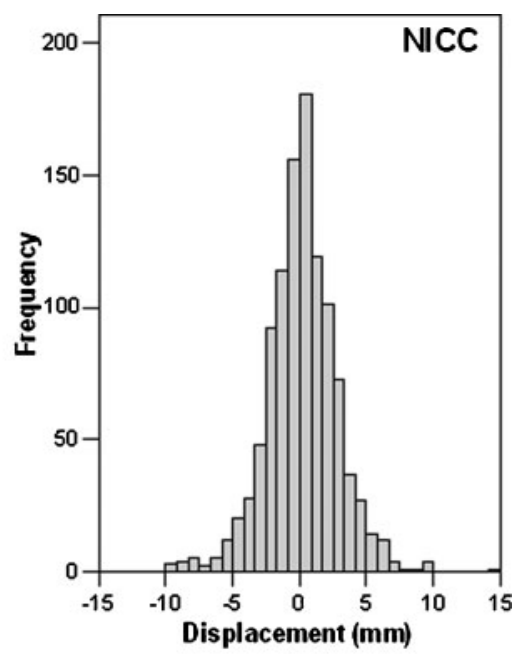

(b)
Figure 1. Histogram of displacements (all directions) at (a) Belvoir Park Hospital (BPH) and (b) Northern Ireland Cancer Centre (NICC). Data are pooled in such a way that a positive value is assigned to a treatment beam being posterior, superior or left with respect to the reference image for the anteroposterior, superior-inferior or lateral displacements, respectively. tion in set-up uncertainties was seen for the lateral direction, and is the direction for which the doctor delineation error and motion contribution were smallest.

Table 4 shows that the margins generated by the van Herk method were similar to those using the BIR method, although they are between $0.8 \mathrm{~mm}$ and $2.7 \mathrm{~mm}$ larger. A further formula suggested by Van Herk et al [23] is:

$$
\text { CTV-PTV margin }=2.5 \sum+0.7 \sigma-3 \mathrm{~mm}
$$

The CTV-PTV margins found using this formula are less than those generated with the BIR method by between $0.3 \mathrm{~mm}$ and $2.2 \mathrm{~mm}$. The use of either Equation 2 or Equation 3 is much simpler than Equation 1, as the planning parameter, algorithm and penumbra errors are not included. The planning parameter is beam orientation dependent and, with the advancement of varying intensity-modulated radiotherapy class solutions, may become more difficult to quantify. Importantly, both Van Herk et al methodologies [3, 23] reveal a margin reduction in the NICC data relative to that of the $\mathrm{BPH}$.

A $10 \mathrm{~mm}$ CTV-PTV margin in all directions for prostate radiotherapy is a widely reported standard [23-28], although some use this margin along with a reduction to $6-8 \mathrm{~mm}$ at the posterior border [29-31]. Margins used in this more recent literature are more consistent than those reported in the review by $\mathrm{Wu}$ et al, [32], where margins ranged from 5.6-17.5 mm, 7-20 mm and $6-20 \mathrm{~mm}$ in the lateral, superior-inferior and AP directions, respectively. At our centre, a margin of $10 \mathrm{~mm}$ is currently used in all directions. The main reason for selecting this margin is its use in clinical trials, such as the RT01 trial, the emerging results of which indicate that this margin is clinically acceptable [33]. Also, it has been postulated [23] that tumour control probability (TCP) does not decrease significantly when reducing margins from $14 \mathrm{~mm}$ to $10 \mathrm{~mm}$. However, a further reduction below $10 \mathrm{~mm}$ may result in a marked decrease in TCP. Hence, the use of $10 \mathrm{~mm}$ margins rather than those calculated will allow a reduction in dose to critical structures while maintaining an acceptable TCP.

Table 2 indicates that there are advantages in using an action level of $5 \mathrm{~mm}$ relative to a protocol of no correction to control systematic set-up uncertainties, particularly in the superior-inferior direction. However, the data seem to imply that there would not be much benefit in reducing the action level further to $3 \mathrm{~mm}$. This may be due to the fact that a $5 \mathrm{~mm}$ action level was not scrupulously observed in practice, with systematic uncertainties of $4 \mathrm{~mm}$ being regularly adjusted at the

Table 2. Systematic and random errors for BPH and NICC (5 mm action level) compared with the example in the BIR (2003) handbook [4] and Enmark et al [19]

\begin{tabular}{|c|c|c|c|c|c|c|}
\hline & \multicolumn{3}{|c|}{ Systematic (mm) } & \multicolumn{3}{|c|}{ Random (mm) } \\
\hline & Lateral & Superior-inferior & Anteroposterior & Lateral & Superior-inferior & Anteroposterior \\
\hline Audit at BPH & 3.3 & 2.4 & 2.7 & 2.5 & 1.7 & 1.8 \\
\hline Audit at NICC & 2.7 & 2.2 & 2.0 & 2.3 & 1.7 & 1.7 \\
\hline BIR (2003) & 1.2 & 3.0 & 1.7 & 2.4 & 2.3 & 2.2 \\
\hline Enmark et al (3DCRT) & 1.5 & 3.4 & 2.1 & 2.6 & 3.7 & 3.0 \\
\hline $\begin{array}{l}\text { NICC with a } \\
\text { protocol of } \\
\text { no correction }\end{array}$ & 2.9 & 2.9 & 2.1 & 2.1 & 1.5 & 1.6 \\
\hline $\begin{array}{l}\text { NICC with } 3 \mathrm{~mm} \\
\text { correction strategy }^{a}\end{array}$ & 2.7 & 2.1 & 2.0 & 2.6 & 1.7 & 1.9 \\
\hline
\end{tabular}

BPH, Belvoir Park Hospital; NICC, Northern Ireland Cancer Centre; 3DCRT, three-dimensional conformal radiotherapy.

aPredicted errors for the NICC data are also shown based on a "a protocol of no correction" and "3 mm correction strategy" simulation. 
Table 3. CTV-PTV margins generated following the BIR method

\begin{tabular}{llll}
\hline & \multicolumn{2}{l}{ CTV-PTV margin $(\mathrm{mm})$} & \\
\cline { 2 - 4 } & Lateral & Superior-inferior & Anteroposterior \\
\hline Audit at BPH & 8.8 & 11.0 & 11.3 \\
Audit at NICC & 8.3 & 11.7 & 11.5 \\
BIR 2003 & 7.2 & 12.2 & 11.2 \\
Enmark et al [19] (3DCRT) & 6.4 & 15.0 & 12.2 \\
\hline
\end{tabular}

CTV, clinical target volume; PTV, planning target volume; BPH, Belvoir Park Hospital; NICC, Northern Ireland Cancer Centre; 3DCRT, three-dimensional conformal radiotherapy.

Table 4. CTV-PTV margins generated following the Van Herk method

\begin{tabular}{llll}
\hline & \multicolumn{2}{l}{ CTV-PTV margin $(\mathrm{mm})$} & Anteroposterior \\
\cline { 2 - 4 } & Lateral & Superior-inferior & 13.7 \\
\hline Audit at BPH & 11.5 & 13.3 & 13.1 \\
Audit at NICC & 10.1 & 13.1 & 12.6 \\
BIR 2003 & 8.8 & 13.3 & 13.4 \\
Enmark et al [19] (3DCRT) & 8.1 & 15.8 & \\
\hline
\end{tabular}

CTV, clinical target volume; PTV, planning target volume; BPH, Belvoir Park Hospital; NICC, Northern Ireland Cancer Centre; 3DCRT, three-dimensional conformal radiotherapy.

Table 5. CTV-PTV margins generated following the BIR method using the original set-up errors (5 mm correction strategy) and simulations of "a protocol of no correction" or "a $3 \mathrm{~mm}$ correction strategy". Also included are margins had no random or systematic set-up errors been present

\begin{tabular}{llll}
\hline & \multicolumn{2}{l}{ CTV-PTV margin $(\mathrm{mm})$} & \\
\cline { 2 - 4 } & Lateral & Superior-inferior & Anteroposterior \\
\hline Simulated at NICC (no CS) & 8.6 & 12.5 & 11.2 \\
Audit at NICC (5 mm CS) & 8.3 & 11.7 & 11.5 \\
Simulated at NICC (3 mm CS) & 8.4 & 11.4 & 11.2 \\
Zero setup/random error & 4.3 & 9.7 & 9.5 \\
\hline
\end{tabular}

CTV, clinical target volume; PTV, planning target volume; NICC, Northern Ireland Cancer Centre; CS, correction strategy.

doctors' discretion. However, it is important to consider that a $3 \mathrm{~mm}$ action level would result in an increase in the number of images acquired during the patients' treatment (to confirm a systematic deviation), as well as an increase in the number of changes made to the patients' set-up instructions (10 of the 25 patients were shifted for the $5 \mathrm{~mm}$ action level compared with 23 of the 25 patients that would have had to be shifted if the $3 \mathrm{~mm}$ action level were observed). This could be analysed more thoroughly by using a $3 \mathrm{~mm}$ action level with a cohort of patients. Nevertheless, Table 5 shows that when systematic or random uncertainties are set to zero, the margins required are still relatively large. This would seem to indicate that changing an action level would have less of an impact than strategies to minimize the doctor delineation or motion uncertainties.

All set-up errors in this work have been attained through analysis of data that utilize an off-line imaging protocol, using bony anatomy rather than the tumour, for image matching. Daily online imaging using softtissue information or seeds should help to reduce margins further $[12,19,32,34-36]$, as the set-up uncertainty and motion error may be contained within a single uncertainty value. Investment in hardware and resources is required to achieve this. Our work suggests that a $3 \mathrm{~mm}$ action level would be of minimal benefit when using off-line bony anatomy-based matching, but this or an even smaller value may be more appropriate for use with an online tumour-based protocol.

\section{Conclusions}

Systematic and random set-up errors, determined from EPIs, have been used to investigate geometric uncertainties of localized prostate cancer radiotherapy. The BIR method includes a number of parameters, some of which must be sourced from published literature. The Van Herk method, although simpler to calculate, was found to generate larger CTV-PTV margins. Margins used clinically still tend to be based on legacy clinical practice or adopted from clinical trial protocols. The acceptability of these in terms of TCP and normal tissue complication probability outcomes does not seem to indicate that margins should be increased based on the margin calculations. However, the greatest advantage in using the calculation techniques may be for monitoring trends in clinical practice or audit changes introduced by new equipment, technology or practice. As such, the choice of the method to use may be less important than using a particular method in a consistent manner.

On re-analysing the EPI data with a simulated $3 \mathrm{~mm}$ action level, the indications were that this reduced 
tolerance would increase workload (through additional imaging and increased adjustment to patient set-up) without realizing a reduction in uncertainties. Therefore, we believe that a $5 \mathrm{~mm}$ action level is adequate for the bony anatomy-based off-line imaging protocol in use at our cancer centre.

\section{Acknowledgment}

The authors wish to acknowledge Dr Mark Grattan for comments on the manuscript.

\section{References}

1. International Commission on Radiation Units and Measurements. ICRU report 50: Prescribing, recording, and reporting photon beam therapy. Bethesda, MD: International Commission on Radiation Units and Measurements, 1993.

2. International Commission on Radiation Units and Measurements. ICRU report 62: Prescribing, recording and reporting photon beam therapy (supplement to ICRU report 50). Bethesda, MD: International Commission on Radiation Units and Measurements, 1999.

3. Van Herk M, Remeijer P, Rasch C, Lebesque JV. Probability of correct dosage: dose-population histograms for deriving treatment margins in radiotherapy. Int J Radiat Oncol Biol Phys 2000;47:1121-35.

4. BIR Working Party. Geometric uncertainties in radiotherapy. London, UK: The British Institute of Radiology, 2003.

5. Van Herk, Errors and margins in radiotherapy. Sem Radiat Oncol 2004;14:52-64.

6. Bijhold J, van Herk M, Vijlbrief R, Lebesque JV. Fast evaluation of patient set-up during radiotherapy by aligning features in portal and simulator images. Phys Med Biol 1991;36:1665-79.

7. Mubata CD, Bidmead AM, Ellingham LM, Thompson V, Dearnaley DP. Portal imaging protocol for radical doseescalated radiotherapy treatment of prostate cancer. Int J Radiat Oncol Biol Phys 1998;40:221-31.

8. Herman MG. Clinical use of electronic portal imaging. Semin Radiat Oncol 2005;15:157-67.

9. Rasch C, Barillot I, Remeijer P, Touw A, van Herk M, Lebesque JV. Definition of the prostate in CT and MRI: a multi-observer study. Int J Radiat Oncol Biol Phys 1999;43:57-66.

10. Langen KM, Jones DT. Organ motion and its management. Int J Radiat Oncol Biol Phys 2001;50:265-78.

11. Wu J, Haycocks T, Alasti H, Ottewell G, Middlemiss N, Abdolell $\mathrm{M}$, et al. Positioning errors and prostate motion during conformal prostate radiotherapy using on-line isocentre set-up verification and implanted prostate markers. Radiother Oncol 2001;61:127-33.

12. Nederveen AJ, Dehnad $H$, van der Heide UA, van Moorselaar RJ, Hofman P, Lagendijk JJ. Comparison of megavoltage position verification for prostate irradiation based on bony anatomy and implanted fiducials. Radiother Oncol 2003;68:81-8.

13. Hoogeman MS, van Herk, de Bois J, Lebesque JV. Stratagies to reduce the systematic error due to tumour and rectum motion in radiotherapy of prostate cancer. Radiotherapy and Oncology 2005;74:177-85.

14. Hurkmans CW, Remeijer P, Lebesque JV, Mijnheer BJ. Set-up verification using portal imaging: review of current clinical practice. Radiother Oncol 2001;58:105-20.

15. Kitamura K, Shirato H, Seppenwoolde Y, Onimaru R, Oda $\mathrm{M}$, Fujita $\mathrm{K}$, et al. Three-dimensional intrafractional movement of prostate measured during real-time tumor-tracking radiotherapy in supine and prone treatment positions. Int J Radiat Oncol Biol Phys 2002;53:1117-23.

16. Malone S, Crook JM, Kendal WS, Szanto J. Respiratoryinduced prostate motion: quantification and characterization. Int J Radiat Oncol Biol Phys 2000;48:105-9.

17. Nichol AM, Brock KK, Lockwood GA, Moseley DJ, Rosewall T, Warde PR, et al. A magnetic resonance imaging study of prostate deformation relative to implanted gold fiducial markers. Int J Radiat Oncol Biol Phys 2007;67:48-56.

18. Schallenkamp JM, Herman MG, Kruse JJ, Pisansky TM. Prostate position relative to pelvic bony anatomy based on intraprostatic gold markers and electronic portal imaging. Int J Radiat Oncol Biol Phys 2005;63:800-11.

19. Enmark E, Korreman S, Nystrom H. IGRT of prostate cancer; is the margin reduction gained from daily IG timedependent? Acta Oncologica 2006;45:907-14.

20. van Herk M, Bruce A, Kroes AP, Shouman T, Touw A, Lebesque JV. Quantification of organ motion during conformal radiotherapy of the prostate by three dimensional image registration. Int J Radiat Oncol Biol Phys 1995;33:1311-20.

21. Bel A, van Herk M, Lebesque JV. Target margins for random geometrical treatment uncertainties in conformal radiotherapy. Med Phys 1996;23:1537-45.

22. Bel A, Vos, PH, Rodrigus PTR, Creutzberg CL, Visser AG, Stroom JC et al. High-precision prostate cancer irradiation by clinical application of an offline patient setup verification procedure, using portal imaging. Int $\mathrm{J}$ Radiation Oncology Biol Phys 1996;35:321-32.

23. Van Herk $M$, Remeijer $P$, Lebesque JV. Inclusion of geometric uncertainties in treatment plan evaluation. Int J Radiat Oncol Biol Phys 2002;52:1407-22.

24. Craig T, Wong E, Bauman G, Battista J, Van Dyk J. Impact of geometric uncertainties on evaluation of treatment techniques for prostate cancer. Int J Radiat Oncol Biol Phys 2005;62:426-36.

25. Peeters ST, Hoogeman MS, Heemsbergen WD, Slot A, Tabak H, Koper PC, et al. Volume and hormonal effects for acute side effects of rectum and bladder during conformal radiotherapy for prostate cancer. Int J Radiat Oncol Biol Phys 2005;63:1142-52.

26. Livsey JE, Wylie JP, Swindell R, Khoo VS, Cowan RA, Logue JP. Do differences in target volume definition in prostate cancer lead to clinically relevant differences in normal tissue toxicity? Int J Radiat Oncol Biol Phys 2004;60:1076-81.

27. Landoni V, Saracino B, Marzi S, Gallucci M, Petrongari MG, Chianese E, et al. A study of the effect of set-up errors and organ motion on prostate cancer treatment with IMRT. Int J Radiat Oncol Biol Phys 2006;65:587-94.

28. Seddon B, Bidmead M, Wilson J, Khoo V, Dearnaley D. Target volume definition in conformal radiotherapy for prostate cancer: quality assurance in the MRC RT-01 trial. Radiother Oncol 2000;56:73-83.

29. Happersett L, Mageras GS, Zelefsky MJ, Burman CM, Leibel SA, Chui C, et al. A study of the effects of internal organ motion on dose escalation in conformal prostate treatments. Radiother Oncol 2003;66:263-70.

30. Bayley AJ, Catton CN, Haycocks T, Kelly V, Alasti H, Bristow $\mathrm{R}$, et al. A randomized trial of supine vs. prone positioning in patients undergoing escalated dose conformal radiotherapy for prostate cancer. Radiother Oncol 2004;70:37-44.

31. Stasi M, Munoz F, Fiorino C, Pasquino M, Baiotto B, Marini $\mathrm{P}$, et al. Emptying the rectum before treatment delivery limits the variations of rectal dose - volume parameters during 3DCRT of prostate cancer. Radiother Oncol 2006;80:363-70.

32. Wu J, Haycocks T, Alasti H, Ottewell G, Middlemiss N, Abdolell M, et al. Positioning errors and prostate motion 
during conformal prostate radiotherapy using on-line isocentre set-up verification and implanted prostate markers. Radiother Oncol 2001;61:127-33.

33. Sydes MR, Stephens RJ, Moore AR, Aird EG, Bidmead AM, Fallowfield LJ, et al. Implementing the UK Medical Research Council (MRC) RT01 trial (ISRCTN 47772397): methods and practicalities of a randomised controlled trial of conformal radiotherapy in men with localised prostate cancer. Radiother Oncol 2004;72:199-211.

34. Van den Heuvel F, Fugazzi J, Seppi E, Forman JD. Clinical application of a repositioning scheme, using gold markers and electronic portal imaging. Radiother Oncol 2006;79: 94-100.

35. Wu Q, Ivaldi G, Liang J, Lockman D, Yan D, Martinez A. Geometric and dosimetric evaluations of an online imageguidance strategy for 3D-CRT of prostate cancer. Int J Radiat Oncol Biol Phys 2006;64:1596-609.

36. Litzenberg DW, Balter JM, Lam KL, Sandler HM, Ten Haken RK. Retrospective analysis of prostate cancer patients with Implanted gold markers using off-line and adaptive Therapy protocols. Int J Radiation Oncology Biol Phys 2005;63:123-33. 